\title{
Probing a single dipolar interaction between a pair of two-level quantum system by scatterings of single photons in an aside waveguide
}

\author{
Xingmin $\mathrm{Li}^{\mathrm{a}}$, L. F. Wei ${ }^{\mathrm{a}, \mathrm{b}, *}$ \\ ${ }^{a}$ State Key Laboratory of Optoelectronic Materials and Technologies, School of Physics and Engineering, \\ Sun Yat-Sen University, Guangzhou 510275, China \\ ${ }^{b}$ Quantum Optoelectronics Laboratory, School of Physical Science and Technology, Southwest Jiaotong \\ University, Chengdu 610031, China
}

\begin{abstract}
Weak dipolar interactions exist widely in various atomic, nuclear and molecular systems, and could be utilized to implement the desired quantum information processings. However, these interactions are relatively weak and hard to be measured precisely. Here, we propose an approach to detect such a weak interaction by probing the transport of a single waveguide-photon scattered by two aside qubits with a single dipolar exchange-interaction. By a full quantum theory of photon transports in optical waveguide, we show that the dipolar interaction between the aside two qubits significantly influence the transmitted spectra of the photon traveling along the one-dimensional waveguide. Thus, probing the relevant changes in the transmitted spectra and the transmission probability distribution specifically for the resonant photons, compared with those scattered by the two individual qubits, the information of the single dipolar interaction between the qubits could be extracted. The feasibility of the proposal is also discussed.
\end{abstract}

Keywords: quantum transmission, dipolar interaction, transmission probability

\footnotetext{
${ }^{*}$ Corresponding author

Email address: weilianfu@gmail.com (L. F. Wei)
}

Preprint submitted to Journal of $\mathrm{BT}_{E} X$ Templates

December 4, 2015

(C) 2015. This manuscript version is made available under the Elsevier user license http://www.elsevier.com/open-access/userlicense/1.0/ 


\section{Introduction}

It is well-known that, due to the wave function overlap, mediated by quantum tunnelling, and through other processes, dipolar interactions widely exist in various nuclear, atomic, and molecular systems [1, 2, 3]. Given these interactions are related to many physical phenomena, e.g., quantum magnetism [4], many-body quantum correlations [5], and quantum computation [6,7], etc., exactly characterizing them are particularly important. Traditionally, this is achieved by detecting the absorption spectra of dipolar-interaction matter radiated by stronger electromagnetic driving [8]. For example, by observing the coherent spin dynamics of the lattice-confined polar molecules or quantum gas, the dipolar spin-exchange interactions between them were successfully measured $[9,10,11,12,13]$. Note that in these demonstrations the collection information of many dipolar interactions, rather than the exact signal of a single dipolar interaction, are extracted. A question is, how to probe a significantly-weak dipolar interaction among few atoms/molecules. This is not trivial as it requires the implementation of very weak electromagnetic signal detections at few-photon level.

In recent years, the development of integrated optoelectronics the detection [14, 15] and manipulation of single photons on a chip $[16,17,18,19,20]$ have been achieved. Quantum interface between the ions and single photons in optical cavity also have been realized in experiments $[21,22]$. These provide a robust way to realize the high quantum efficiency photon detection [14, 23], since the photon is now confined in a waveguide. Based on these developments, we propose here an approach to realize the weak dipolar interaction between two two-level atoms (i.e., qubits) by probing the single-photon transports along a one-dimension waveguide [24]. In fact, controllable single-photon transports along one-dimensional waveguide have been extensively studied $[16,17,18,19,20]$. One of the main results in these works is, the atom-photon interaction can be utilized to control the transmission and reflection spectra of the photon transporting along the waveguide. Naturally, the scatter from the dipolar-interaction matter of the photon in the waveguide changes the spectra of the transmitted photons. As a consequence, a single dipolar interaction between the two scatters could be detected by probing the relevant spectral changes. Specifically, the existence of the 
scatters should modify the transmission probability of the incident resonant photons for different phase shifts.

The paper is organized as follows. In Sec. 2 we introduce our model, in the framework of a full quantum scattering theory, to treat the transports of single photons along a one-dimensional waveguide scattered by a pair of two-level atoms. The transmission and reflection probabilities of the single photons are specifically analyzed in the Sec. 3. We investigate analytically and numerically how the dipolar interaction between the qubits influence the transmission spectra of the photons and also the phaseshift-dependent transmission probability distributions of the incident resonant photons. Compared with those for two individual qubits as the scatters, the existing dipolar interaction between the two qubits can be extracted. Finally, we summarize our results in Sec. 4 and discuss the feasibility of the present proposal.

\section{Scatterings of single photons by a pair of qubits aside a one-dimensional waveg- uide}

We consider the configuration shown in Fig. 1, wherein a single photon propagates along a one-dimensional optical waveguide scattered by two two-level atoms encoded as the qubits. Generically, the dipolar interaction between the atoms can be written as

$$
H_{d i p}=-\frac{\mu_{0}}{4 \pi r_{12}^{3}}\left[3\left(\mathbf{m}_{1} \cdot \mathbf{e}_{12}\right)\left(\mathbf{m}_{2} \cdot \mathbf{e}_{12}\right)-\mathbf{m}_{1} \cdot \mathbf{m}_{2}\right],
$$

with $\mu_{0}$ being the magnetic constant and $\mathbf{m}_{j}$ being the magnetic moment of the $j$-th atom. $r_{12}$ is the distance between the two atoms with $\mathbf{e}_{12}$ being the unit vector parallel to the line joining the centers of the two atoms. Under the usual rotating-wave

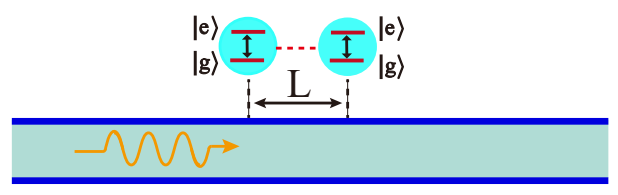

Figure 1: Single photon transports along a one-dimensional waveguide scattered by two aside two-level atoms (separated by $L$ ). 
approximation the above dipolar interaction can be simplified as

$$
H_{\text {dip }}=g\left(\sigma_{1}^{+} \sigma_{2}^{-}+\sigma_{2}^{+} \sigma_{1}^{-}\right) \text {, }
$$

with the dipolar interaction strength $g=-\mu_{0} \hbar^{2} \gamma_{1} \gamma_{2}\left(1-3 \cos ^{2} \Theta\right) /\left(16 \pi r_{12}^{3}\right)$. Here, $\gamma_{j}$ is the gyromagnetic ratios of the $j$-th atom, $\Theta$ is the angle between the quantization axis of the both atoms and the direction of the spins, and $\sigma_{j}^{+}$and $\sigma_{j}^{-}$the $j$-th atomic raising and lowering ladder operators, respectively. Above, the usual rotating-wave approximation has been used. Therefore, the single photon propagating in the waveguide scattered by the two atoms can be described by the following Hamiltonian $(\hbar=1)$ [24]

$$
\begin{aligned}
H= & \sum_{j=1,2}\left(\Omega_{j}-\frac{i \Gamma_{j}}{2}\right) \sigma_{j}^{+} \sigma_{j}^{-}+g\left(\sigma_{1}^{+} \sigma_{2}^{-}+\sigma_{2}^{+} \sigma_{1}^{-}\right) \\
& +\int \frac{c}{i} d x\left[a_{R}^{\dagger}(x) \frac{\partial}{\partial x} a_{R}(x)-a_{L}^{\dagger}(x) \frac{\partial}{\partial x} a_{L}(x)\right] \\
& +\sum_{j=1,2} \int d x V_{j} \delta\left(x-l_{j}\right)\left[a_{R}^{\dagger}(x) \sigma_{j}^{-}+a_{L}^{\dagger}(x) \sigma_{j}^{-}+H . c .\right] .
\end{aligned}
$$

Here, $\Omega_{j}$ and $\Gamma_{j}$ are the $j$-th atomic transition frequency and dissipation rate, respectively. $l_{j}$ is the position of the $j$-th atom and $L=l_{2}-l_{1}$ the distance between the atoms. Also, $a_{R}^{\dagger}(x)\left(a_{R}(x)\right)$ and $a_{L}^{\dagger}(x)\left(a_{L}(x)\right)$ are the creation (annihilation) operators of the photon propagating right and left, respectively. $c$ is the group velocity of the photon traveling along the waveguide. $V_{j}$ is the coupling strength between the photon and the $j$-th atom.

Suppose a single photon is incident from the left direction. Then, the stationary solution of the system can be obtained by solving the time-independent Schrödinger equation

$$
H|\Phi\rangle=E|\Phi\rangle
$$

where $E$ is the eigenvalue, and

$$
|\Phi\rangle=\int d x\left[\phi_{R}(x) a_{R}^{\dagger}(x)+\phi_{L}(x) a_{L}^{\dagger}(x)\right]|\varnothing\rangle+\left(e_{1} \sigma_{1}^{+}+e_{2} \sigma_{2}^{+}\right)|\varnothing\rangle
$$

is the generic stationary wave function [19] of the system. Here, $|\varnothing\rangle$ denotes the vacuum state without any photon in the waveguide and all the atoms are in their ground 
states. $\phi_{R / L}$ and $e_{j}$ stand for the probabilistic amplitudes of the photon propagating along the $R / L$ direction and the excitation of the $j$-th atom, respectively.

Substituting Eq. 5 into Eq. 4, the coefficients in Eq. 4 are determined by

$$
\begin{aligned}
E \phi_{R}(x) & =\frac{c}{i} \frac{\partial}{\partial x} \phi_{R}(x)+V_{1} \delta\left(x-l_{1}\right) e_{1}+V_{2} \delta\left(x-l_{2}\right) e_{2} \\
E \phi_{L}(x) & =\frac{-c}{i} \frac{\partial}{\partial x} \phi_{L}(x)+V_{1} \delta\left(x-l_{1}\right) e_{1}+V_{2} \delta\left(x-l_{2}\right) e_{2} \\
E e_{1} & =V_{1} \phi_{R}\left(l_{1}\right)+V_{1} \phi_{L}\left(l_{1}\right)+\left(\Omega_{1}-\frac{i \Gamma_{1}}{2}\right) e_{1}+g e_{2} \\
E e_{2} & =V_{2} \phi_{R}\left(l_{2}\right)+V_{2} \phi_{L}\left(l_{2}\right)+\left(\Omega_{2}-\frac{i \Gamma_{2}}{2}\right) e_{2}+g e_{1} .
\end{aligned}
$$

Furthermore, assume that the wave vector of the photon is $k$ and the $j$-th atom is located at $l_{j}$ aside the waveguide. Then, the coefficients $\phi_{R}(x)$ and $\phi_{L}(x)$ can be rewritten as [19]

$$
\begin{gathered}
\phi_{R}(x)=\frac{e^{i k x}}{\sqrt{2 \pi}}\left[\theta\left(l_{1}-x\right)+t_{12} \theta\left(x-l_{1}\right) \theta\left(l_{2}-x\right)+t \theta\left(x-l_{2}\right)\right], \\
\phi_{L}(x) \quad=\frac{e^{-i k x}}{\sqrt{2 x}}\left[r \theta\left(l_{1}-x\right)+r_{12} \theta\left(x-l_{1}\right) \theta\left(l_{2}-x\right)\right],
\end{gathered}
$$

where $\theta(x)$ is the step function, $t_{12}(r)$ and $t\left(r_{12}\right)$ are the transmission (reflection) amplitudes at $l_{1}$ and $l_{2}$, respectively. Substituting Eqs. 10- 11 into Eqs. 6-9 and setting $\theta(0)=1 / 2$ for simplicity, the reflection and transmission amplitudes of the traveling photon are obtained as

$$
\begin{gathered}
r=\frac{-\frac{i V_{2}^{2}}{c} e^{2 i k l_{2}}\left(E-\Omega_{1}+i \frac{\Gamma_{1}}{2}-i \frac{V_{1}^{2}}{c}\right)-i \frac{V_{1}^{2}}{c} e^{2 i k l_{1}}\left(E-\Omega_{2}+i \frac{\Gamma_{2}}{2}+i \frac{V_{2}^{2}}{c}\right)-2 i g \frac{V_{1} V_{2}}{c} e^{i k\left(l_{1}+l_{2}\right)}}{\prod_{j=1,2}\left(E-\Omega_{j}+i \frac{\Gamma_{j}}{2}+i \frac{V_{j}^{2}}{c}\right)-\left[i \frac{V_{2}^{2}}{c} e^{2 i k\left(l_{2}-l_{1}\right)}-\frac{g V_{2}}{V_{1}} e^{i k\left(l_{2}-l_{1}\right)}\right]\left[i \frac{V_{1}^{2}}{c}-\frac{g V_{1}}{V_{2}} e^{-i k\left(l_{2}-l_{1}\right)}\right]}, \\
t=\frac{\left(E-\Omega_{1}+i \frac{\Gamma_{1}}{2}\right)\left(E-\Omega_{2}+i \frac{\Gamma_{2}}{2}\right)+i g \frac{V_{1} V_{2}}{c} e^{i k\left(l_{2}-l_{1}\right)}-i g \frac{V_{1} V_{2}}{c} e^{-i k\left(l_{2}-l_{1}\right)}-g^{2}}{\prod_{j=1,2}\left(E-\Omega_{j}+i \frac{\Gamma_{j}}{2}+i \frac{V_{j}^{2}}{c}\right)-\left[i \frac{V_{2}^{2}}{c} e^{2 i k\left(l_{2}-l_{1}\right)}-\frac{g V_{2}}{V_{1}} e^{i k\left(l_{2}-l_{1}\right)}\right]\left[i \frac{V_{1}^{2}}{c}-\frac{g V_{1}}{V_{2}} e^{-i k\left(l_{2}-l_{1}\right)}\right]},
\end{gathered}
$$

with $E=k c$. Specifically, if the atoms are identical and also ideal (without any dissipation), i.e., $\Gamma_{1}=\Gamma_{2}=\Gamma \sim 0, \Omega_{1}=\Omega_{2}=\Omega$ and $V_{1}=V_{2}=V$, then the above equations reduce to

$$
\begin{aligned}
& t=\frac{\Delta^{2}+i g \frac{\gamma}{2} e^{i \theta}-i g \frac{\gamma}{2} e^{-i \theta}-g^{2}}{\left(\Delta+i \frac{\gamma}{2}\right)^{2}-\left(i \frac{\gamma}{2} e^{2 i \theta}-g e^{i \theta}\right)\left(i \frac{\gamma}{2}-g e^{-i \theta}\right)}, \\
& r=\frac{-i \frac{\gamma}{2} e^{i \theta}\left(\Delta-i \frac{\gamma}{2}\right)-i \frac{\gamma}{2}\left(\Delta+i \frac{\gamma}{2}\right)-i g \gamma e^{i \theta}}{\left(\Delta+i \frac{\gamma}{2}\right)^{2}-\left(i \frac{\gamma}{2} e^{2 i \theta}-g e^{i \theta}\right)\left(i \frac{\gamma}{2}-g e^{-i \theta}\right)} .
\end{aligned}
$$

Here, $\Delta=E-\Omega$ is the detuning, $\gamma=2 V^{2} / c$ is the effective coupling strength between the atoms and the photon in the waveguide. $\theta=k L$ is the phase shift. Above, we also let $l_{1}=0$ and $l_{2}=L$ for simplicity. 
A measurable quantify of the photon transporting in the system is the transmission probability

$$
T=|t|^{2}=\frac{\left(\frac{\Delta^{2}-g^{2}}{\gamma^{2}}-\frac{g}{\gamma} \sin \theta\right)^{2}}{\left(\frac{\Delta^{2}-g^{2}}{\gamma^{2}}-\frac{\sin ^{2} \theta}{2}-\frac{g}{\gamma} \sin \theta\right)^{2}+\left(\frac{\Delta+g \cos \theta}{\gamma}+\frac{\sin 2 \theta}{4}\right)^{2}} .
$$

In the following, we will discuss how to characterize the dipolar interaction between the two-level atoms by investigating the transmission spectra of the photon scattered by the qubits, with and without the dipolar interaction, respectively.

\section{Probing the dipolar interaction between a pair of qubits}

With the transmission probability demonstrated above for the photon transporting along the waveguide scattered by the aside two qubits, we now discuss how the interaction of the qubits can be probed. This can be achieved by measuring the transmitted behaviors, such as the frequency-dependent transmission probability and the phase shift of the resonant photons.

\subsection{The existing dipolar interaction between the qubits influence the transmission} spectra of the waveguide photons

First, for two individual qubits without the dipolar interaction, i.e., $g=0$, the transmission spectrum is similar to the Fig.1(b) in Ref. [19]. It is seen that: i)The resonant photon (i.e., the frequency of the incident equals to the eigenfrequency of the qubits)

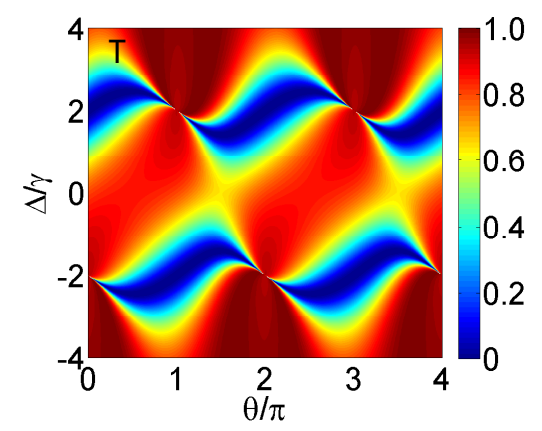

Figure 2: Single-photon transmission spectra scattered by two qubits with the dipolar interaction $g=2 \gamma$. 
is reflected completely. With the increase of the detuning, the reflected (transmitted) probability of the photon decreases (increases). Consequently, the spectrum exhibits a large area of perfect transmission, i.e., for the large detuning limit the photon transmits perfectly; ii)The transmission spectrum is a periodic function of the phase $\operatorname{shift} \theta$, with the period of $\pi$. Comparably, Fig. 2 shows that, in the presence of dipolar interaction between the qubits, e.g., $g=2 \gamma$, the relevant spectrum of the photon transmission reveals certain different features: a)The resonant photon (with $\Delta=0$ ) can transmit with certain probability, rather than be reflected completely as shown in Ref. [19]. This is because that the dipolar interaction between the qubits changes the energy structure of the two-qubit scatter, i.e., the present scatter is not resonance with the incident photons. b)The period of the transmission probability $T$, depending on the phase shift, changes to be $2 \pi$. With these spectral changes, the dipolar interaction between the qubits can be determined.

Specifically, for the incident photons with sufficiently-long wavelength, i.e., $\theta=$ $k L=2 \pi L / \lambda \ll \pi$, and thus $\sin \theta \sim 0$, the transmission amplitude Eq. 14 reduces to

$$
t=\frac{\Delta-g}{\Delta-g+i \gamma} .
$$

This indicates that, at the complete reflection point $T=0$, i.e., the dip of the transmission spectrum of the photon, the measured value of the detuning $\Delta$ is just equivalent to the dipolar interaction strength $g$. This is more clearly shown by Fig. 3(a), wherein the detuning-dependent transmission probability is schematized for $\theta=0$. Indeed, compared with the completely-reflected point at $\Delta=0$ for the two individual qubits, the dipolar interaction between the qubits shifts the completely reflected point into the point $\Delta=g$. Therefore, by probing the transmitted dip (i.e., $T=0$ ), the dipolar interaction between the qubits can be measured as $g=|\Delta|=|\omega-\Omega|$, with $\omega$ being the frequency of the incident photon. More generically, for the relatively-short wavelength of the incident photon (i.e., the phase shift $\theta=k L=\omega L / c$ is definite), we can still extract the dipolar interaction information between the qubits by probing the location(s) of the transmitted dip(s) in the spectra shown by, e.g., Fig. 3(b). Indeed, Eq. 16 implies that, if $\sin \theta \neq 0$, then the transmitted spectra show two-dip structure with the dips being determined as $\Delta^{2}-g^{2}-g \gamma \sin \theta=0$. Suppose that the coupling strength $V$ be- 

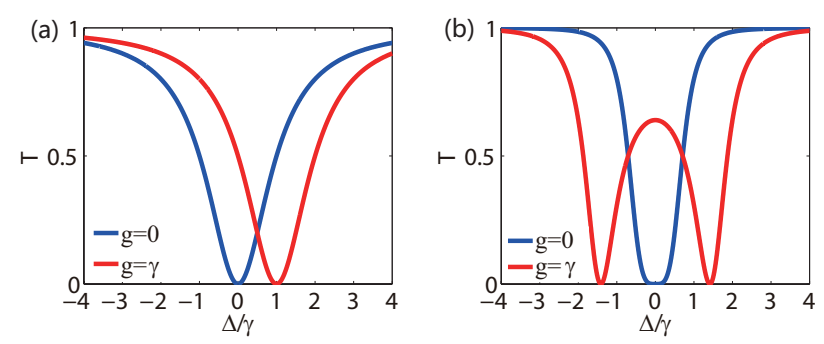

Figure 3: Single-photon transmission spectra for different phase shifts: (a) $\theta \approx 0$, and (b) $\theta=k L=\pi / 2$. When $g=0$, the spectra always shows a single-dip structure; When $g \neq 0$, double-dip structure could be observed in the spectra.

tween the photon and qubits is unchanged for the different frequencies of the incident photons, i.e., $\gamma=2 V^{2} / c$ can be regarded as a constant. Then, the probed locations of the two dips satisfy the following equations

$$
\begin{aligned}
& \left(\omega_{1}-\Omega\right)^{2}-g \gamma \sin \left(k_{1} L\right)-g^{2}=0, \\
& \left(\omega_{2}-\Omega\right)^{2}-g \gamma \sin \left(k_{2} L\right)-g^{2}=0,
\end{aligned}
$$

with $k_{1}=\omega_{1} / c$ and $k_{2}=\omega_{2} / c$ being the wave vectors of the incident photons at transmitted dips, respectively. Solving these equations extracted from the observed transmitted dips, the dipolar interaction strength $g$ and the distance $L$ between the qubits can also be obtained.

\subsection{Probing the dipolar interaction between two atoms with the incident resonant photons}

Specifically, for the resonant incident photon (i.e., $\Delta=0$ ), the transmission amplitude Eq. 14 reduces to

$$
t=\frac{i g \frac{\gamma}{2} e^{i \theta}-i g \frac{\gamma}{2} e^{-i \theta}-g^{2}}{-\frac{\gamma^{2}}{4}-\left(i \frac{\gamma}{2} e^{2 i \theta}-g e^{i \theta}\right)\left(i \frac{\gamma}{2}-g e^{-i \theta}\right)} .
$$

It is seen schematically in Fig. 4(a) that, depending on the phase shift the transmission probability of the resonant incident photon is also changed by the existence of the dipolar interaction between the qubits. Obviously, the transmission probability distribution of the resonant photons is divided into two parts, besides the phase $\theta=\pi$. 

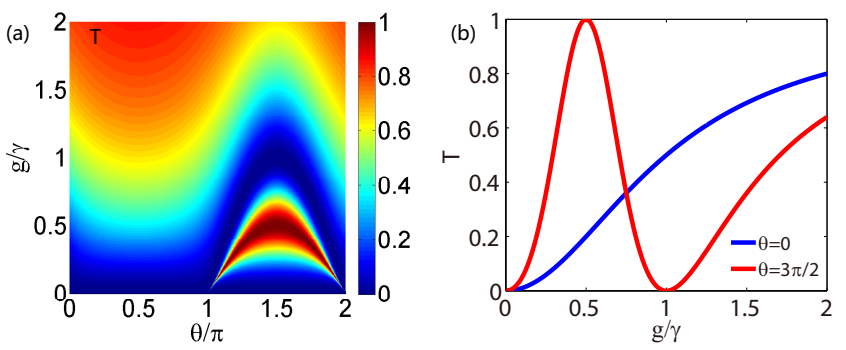

Figure 4: (a) The transmission probability versus the dipolar interaction strength $g$ between the qubits of the resonant incident photons (i.e., $\Delta=0$ ) for different the phase shift $\theta$. (b) The transmission probability of the resonant photons versus the parameter $g$ for the specific phase shifts: $\theta=0$ (blue) and $\theta=3 \pi / 2$ (red).

Fig. 4(b) specifically shows that, for the sufficiently short distance between the qubits (i.e., $L / \lambda=\theta \sim 0$ ), the transmission probability increases with the increase of the dipolar interaction strength $g$ (the blue line). Alternatively, if the distance between the two qubits is sufficiently long, i.e., the induced phase shift $\theta>\pi$, the transmission probability distribution of the resonant photons is relatively complicated. For example, for a defined phase shift $\theta=3 \pi / 2$ the transmission curve, shown the red line in Fig. 4(b), the resonant photons have two complete reflection points; locating at $g / \gamma=0$ and $g / \gamma=1$, respectively. However, in the phase shift $\theta$ ranges $(\pi, 2 \pi)$, two complete transmission points are always found. For example, the complete reflected points are found at $g / \gamma=0$ and $g / \gamma=1$ for the phase shift $3 \pi / 2$. Therefore, by probing the phase-shift-dependent complete reflection points of the resonant photons, the dipolar interaction $g$ between the qubits can also be detected. This offers another approach to measure the dipolar interaction between the two qubits separated by a relatively long distance.

\section{Summary}

In summary, we have investigated how to detect a single dipolar interaction between two qubits by probing the transmission properties of the photon transporting along the aside waveguide. It is shown that the transmission spectra of the photon scattered by the two qubits aside the waveguide are different for the presence/absence of the dipolar interaction between the qubits. Specifically, the resonant photon (i.e., its frequency is 
equivalent to that of the qubit) is completely reflected by two qubits without dipolar interaction, but could be transmitted (with certain probability) if the dipolar interaction exists. In particular, if the phase shift is negligible due to the sufficiently long wave function (compared with the distance between the qubits), then the existence of the dipolar interaction between the qubits simply shift the transmitted dip of the photon from $\Delta=0$ to $|\Delta|=g$, the strength of the dipolar interaction. More generically, if the phase shift $\theta$ satisfies the condition $\sin \theta \neq 0$, then the existence of the dipolar interaction between the qubits yields a two-dip structure in the transmitted spectra of the photon. As a consequence, by probing the locations of the two dips, the strength $g$ of the dipolar interaction can be measured. Specifically, for the resonant incident photon the transmission probability will be increased with the dipolar interaction strength within the phase shift ranges: from $\theta=0$ to $\theta=\pi$. For the other range of the phase shifts (e.g., from $\theta=\pi$ to $\theta=2 \pi$ ), the photon can be either completely reflected or complete transmission, depending on the specifical phase shifts. This provides another effectively approach to probe the dipolar interaction experimentally by measuring the complete reflection points of the resonant incident photons.

In principle, our proposal is feasible for various atom-waveguide systems with the atomic dipolar interactions, e.g., the natural atoms (e.g., ions) coupled to optical waveguides or artificial atoms (e.g., quantum dots and superconducting Josephson circuits) coupled to microwave transmission lines. In fact, in the experiment [9], wherein the eigenfrequency of each qubits is $\Omega=2 \pi \times 2.2 \mathrm{GHz}$ and the dipolar interaction strength between them is $g=2 \pi \times 52 \mathrm{~Hz}$ for the qubit-qubit distance $L=532 \mathrm{~nm}$, the shift $|\Delta|=g$ of the transmitted dip of the resonant photons is obviously measurable. Here, due to the significantly long wave length of the incident photons and the very short distance between the qubits, i.e., $L / \lambda \sim 0$ and $\sin \theta=0$, the observable dip shift is insensitive to the photon-qubit coupling. Similarly, for the off-resonant incident photons the double dips could also be characterized. Thus, a single dipolar interaction between the present two two-level atoms, rather than the collection of many dipolar interactions between the atoms in either optical lattices $[9,10,11,12]$ or quantum gas [13], can be probed with the current spectral technique, at least theoretically. 


\section{Acknowledgements}

This work was supported in part by the National Science Foundation grant Nos. 11174373, 61301031, and U1330201.

\section{References}

[1] K.-K. Ni, S. Ospelkaus, M. H. G. de Miranda, A. Péer, B. Neyenhuis, J. J. Zirbel, S. Kotochigova, P. S. Julienne, D. S. Jin, J. Ye, Science 322 (2008) 231-235.

[2] S. Ospelkaus, K.-K. Ni, D. Wang, M. H. G. de Miranda, B. Neyenhuis, G. Quéméner, P. S. Julienne, J. L. Bohn, D. S. Jin, J. Ye, Science 327 (2010) 853857.

[3] Stefano Giovanazzi, Axel Görlitz, Tilman Pfau, Phys. Rev. Lett. 89 (2002) 130401 .

[4] Ryan Barnett, Dmitry Petrov, Mikhail Lukin, Eugene Demler, Phys. Rev. Lett. 96 (2006) 190401.

[5] A. Micheli, G. K. Brennen, P. Zoller, Nat. Phys. 2 (2006) 341-347.

[6] M. D. Lukin, M. Fleischhauer, R. Cote, L. M. Duan, D. Jaksch, J. I. Cirac, P. Zoller, Phys. Rev. Lett. 87 (2001) 037901.

[7] D. DeMille, Phys. Rev. Lett. 88 (2002) 067901.

[8] E. R. Andrew, Nuclear Magnetic Resonance, Cambridge, 2009.

[9] Bo Yan, Steven A. Moses, Bryce Gadway, Jacob P. Covey, Kaden R. A. Hazzard, Ana Maria Rey, Deborah S. Jin, Jun Ye, Nature 501 (2013) 521-525.

[10] S. Trotzky, P. Cheinet, S. Fölling, M. Feld, U. Schnorrberger, A. M. Rey, A. Polkovnikov, E. A. Demler, M. D. Lukin, I. Bloch, Science 319 (2008) 295-299.

[11] Daniel Greif, Thomas Uehlinger, Gregor Jotzu, Leticia Tarruell, Tilman Esslinger, Science 340 (2013) 1307-1310 
[12] Marco Anderlini, Patricia J. Lee, Benjamin L. Brown, Jennifer Sebby-Strabley, William D. Phillips, J. V. Porto, Nature 448 (2007) 452-456.

[13] J. Stuhler, A. Griesmaier, T. Koch, M. Fattori, T. Pfau, S. Giovanazzi, P. Pedri, L. Santos, Phys. Rev. Lett. 95 (2005) 150406.

[14] G. Romero, J. J. García-Ripoll, E. Solano, Phys. Rev. Lett. 102 (2009) 173602.

[15] Y.-F. Chen, D. Hover, S. Sendelbach, L. Maurer, S. T. Merkel, E. J. Pritchett, F. K. Wilhelm, R. McDermott, Phys. Rev. Lett. 107 (2011) 217401.

[16] A. Wallraff, D. I. Schuster, A. Blais, L. Frunzio, R.- S. Huang, J. Majer, S. Kumar, S. M. Girvin, R. J. Schoelkopf, Nature 431 (2004) 162-167.

[17] Jung-Tsung Shen, Shanhui Fan, Phys. Rev. A 79 (2009) 023837.

[18] W. Z. Jia, Z. D. Wang, Phys. Rev. A 88 (2013) 063821.

[19] Huaixiu Zheng, Harold U. Baranger, Phys. Rev. Lett. 110 (2013) 113601.

[20] Jung-Tsung Shen, Shanhui Fan, Phys. Rev. Lett. 95 (2005) 213001.

[21] L. Lamata, D. R. Leibrandt, I. L. Chuang, J. I. Cirac, M. D. Lukin, V. Vuletić, S. F. Yelin, Phys. Rev. Lett. 107 (2011) 030501.

[22] B. Casabone, K. Friebe, B. Brandstätter, K. Schüppert, R. Blatt, T. E. Northup, Phys. Rev. Lett. 114 (2015) 023602.

[23] B. Peropadre, G. Romero, G. Johansson, C. M. Wilson, E. Solano, J. J. GarcíaRipoll, Phys. Rev. A 84 (2011) 063834.

[24] Dibyendu Roy, Sci. Rep. 3 (2013) 2337. 